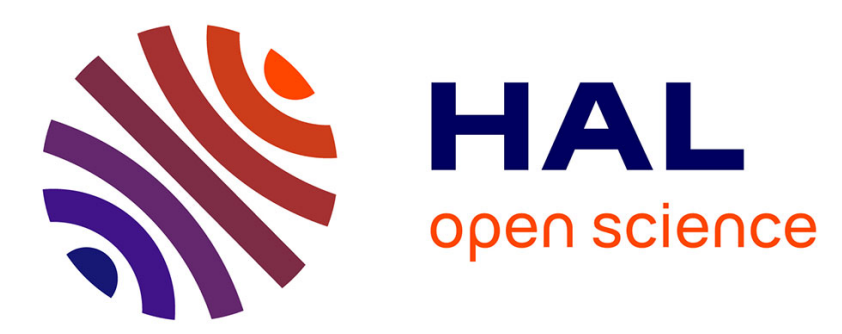

\title{
Methodology for Automatic Movement Cycle Extraction Using Switching Linear Dynamic System
}

Roberto de Souza Baptista, Antonio Padilha Lanari Bo, Mitsuhiro Hayashibe

\section{To cite this version:}

Roberto de Souza Baptista, Antonio Padilha Lanari Bo, Mitsuhiro Hayashibe. Methodology for Automatic Movement Cycle Extraction Using Switching Linear Dynamic System. NER: Neural Engineering, Apr 2015, Montpellier, France. pp.743-746, 10.1109/NER.2015.7146730 . lirmm-01235860

\section{HAL Id: lirmm-01235860 \\ https://hal-lirmm.ccsd.cnrs.fr/lirmm-01235860}

Submitted on 25 Jun 2019

HAL is a multi-disciplinary open access archive for the deposit and dissemination of scientific research documents, whether they are published or not. The documents may come from teaching and research institutions in France or abroad, or from public or private research centers.
L'archive ouverte pluridisciplinaire HAL, est destinée au dépôt et à la diffusion de documents scientifiques de niveau recherche, publiés ou non, émanant des établissements d'enseignement et de recherche français ou étrangers, des laboratoires publics ou privés. 


\title{
Methodology for Automatic Movement Cycle Extraction using Switching Linear Dynamic System
}

\author{
Roberto de Souza Baptista, Antônio Padilha Lanari Bó and Mitsuhiro Hayashibe
}

\begin{abstract}
Human motion assessment is key for motorcontrol rehabilitation. Using standardized definitions and spatiotemporal features - usually presented as a movement cycle diagram- specialists can associate kinematic measures to progress in rehabilitation therapy or motor impairment due to trauma or disease. Although devices for capturing human motion today are cheap and widespread, the automatic interpretation of kinematic data for rehabilitation is still poor in terms of quantitative performance evaluation. In this paper we present an automatic approach to extract spatiotemporal features from kinematic data and present it as a cycle diagram. This is done by translating standard definitions from human movement analysis into mathematical elements of a Switching Linear Dynamic System model. The result is a straight-forward procedure to learn a tracking model from a sample execution. This model is robust when used to automatically extract the movement cycle diagram of the same motion (the Sit-StandSit, as an example) executed in different subject-specific manner such as his own motion speed.
\end{abstract}

\section{INTRODUCTION}

Human motion assessment is key in motor-control rehabilitation. Biomechanicists analyse kinematic measurements using standard definitions to point out key events (the instant of change in the orientation of the movement pattern) and divide each motion type in components and phases according to spatiotemporal features. This type of description - usually presented as a movement cycle diagram- facilitates the collection of normative data for each motion type across different populations. The standardized features and normative data are used to monitor a patient's progress and investigate the effects of trauma or diseases in motor function capability [1], [2], [3].

Automatic human motion tracking systems proliferated in the last decades. From the gold standard multi-infraredcamera systems to the low cost portable systems, such as the Microsoft Kinect or inertial sensors, obtaining precise kinematic human data today is affordable and widespread [3], [4]. However, the post-processing techniques for automatic spatiotemporal feature extraction from kinematic data are still emerging.

In the context of human motion segmentation and classification for rehabilitation, an important distinction must

*This work was partially funded by CAPES - Coordenação de Aperfeiçoamento de Pessoal de Nível Superior

R.S. Baptista is with LARA, Universidade de Brasília, Brazil and INRIA DEMAR Project and LIRMM, University of Montpellier, France baptista@ieee.org

A.P.L. Bó is with LARA, Universidade de Brasília, Brazil antonio.plbelara.unb.br

M. Hayashibe is with INRIA DEMAR Project and LIRMM, CNRS/University of Montpellier, France hayashibed lirmm. fr be made about the meaning of the task of segmentation and classification. One problem is to segment a sequence of unknown movements into single executions followed by the classification of movement type (labelling each single execution according to a set of possible candidates), which has been recently investigated with important results such as done by Lin et al.[5] and Dios et al. [6]. Another problem is: given a single execution (or a repetitive sequence) of a known movement type (a sequence of steps, or a sequence of sit-stand-sit movements), pinpoint the key events in order to extract useful information, i.e. spatiotemporal features. This paper deals with the latter .

Regarding problem of pinpointing events in a known movement type the few current solutions are specifically designed for each application: they depend on the type of sensor, on the motion executed, and/or ignore standard biomechanics descriptions [3], [7]. These approaches limit the use of normative data and results from previous studies to assess the quality of the motion. Besides, performance comparison among techniques is impossible because of their specificity.

This paper presents a methodology to automatically extract spatiotemporal features from a set of kinematic measurements of a previously known human motion using Switching Linear Dynamic System(SLDS) modelling. a technique used to represent complex, non-linear systems through a combination of simpler linear models [8], [9]. SLDS is useful for modelling real world processes that exhibit multiples types of behaviour, - which is particularly true for human motion and has been successfully applied to model human activities, such as the detection of sleep apnea based on breathing patterns during sleep [9] and distinguishing between gait and jogging sequences based on video analysis[8].

In this paper we propose an automatic movement cycle extraction with SLDS by taking motion example from the Sit-Stand-Sit movement.

\section{Methodology}

\section{A. Standard Definitions}

In this work we aim to automatically extract a movement cycle diagram using Switching Linear Dynamic Systems modelling and the standard definitions for movement analysis (with the Sit-Stand-Sit motion as an example).We take the definitions used by [10] to describe the Sit-Stand-Sit movement:

- Events (e) - each event is a single identifiable occurrence, characterized by a marked change in the orientation of the recorded movement pattern. 
- Components (c) - are defined as those constituent parts of the movement, that are bounded by events within the same variable.

- Phases - are collections of components and are also bounded by events, but the boundaries can be established using events from different components.

- Movement cycle - is a sequence of one occurrence of all phases in the movement.

Note that some movements, such as only the Sit to Stand transition, are non-cyclic (movements with distinct starting and ending positions) and therefore a sequence of movement cycles is impossible. Although we are using as an example the Sit-Stand-Sit movement, which is cyclic, the proposed approach is also suited for non-cyclic movements.

\section{B. Switching Linear Dynamic Systems}

Switching Linear Dynamic System - also called in the literature Switching State-Space Models, Switching Kalman Filter Models or Jump-Markov Model- is a technique used to represent complex, non-linear systems through a combination (or switching) of simpler linear state-space models.

It is a state-space model in the form:

$$
\begin{aligned}
x_{t+1} & =A\left(s_{t+1}\right) x_{t}+v_{t+1}\left(s_{t+1}\right) \\
y_{t} & =C x_{t}+w_{t}, \quad \text { with } \\
x_{0} & =v_{0}\left(s_{0}\right)
\end{aligned}
$$

where $x_{t} \in \Re^{N}$ is the hidden state of the state-space model, $v_{t}$ is the state noise, $y_{t} \in \Re^{M}$ is the observed measurement of the system, $w_{t}$ is the measurement noise. $A\left(s_{t}\right)$ is the state transition matrix and $C$ is the observation matrix, as in a conventional linear dynamic system state-space model.

The switching variable, $s_{t}$, belongs to a set of $S$ discrete symbols $\left\{c_{1}, \ldots, c_{S}\right\}$ and its process evolves in time according to the model:

$$
\begin{aligned}
\operatorname{Pr}\left(s_{t+1} \mid s_{t}\right) & =s_{t+1}^{\prime} \Pi s_{t}, \text { with } \\
\operatorname{Pr}\left(s_{0}\right) & =\pi_{0}
\end{aligned}
$$

where the state transition matrix $\Pi$, whose elements are $\Pi(i, j)=\operatorname{Pr}\left(s_{t+1}=c_{i} \mid s_{t}=c_{j}\right)$, represents the probability of $s_{t+1}=c_{i}$, given that $s_{t}=c_{j}$. The state transition matrix $A\left(s_{t}\right)$ and the measurement noise $v\left(s_{t}\right) \sim N\left(0, Q\left(s_{t}\right)\right)$ are associated with a switching variable $s_{t}$, which indicates which model $\left(A\left(s_{t}\right), v_{t}\right)$ is used at each time $t$. The Switching Linear Dynamic System approach develops the probabilistic equations for learning the parameters of the models (specially $A\left(c_{S}\right), Q\left(c_{S}\right), \Pi$ ) and tracking of the observed measurements in a time-series (specially $s_{t}, x_{t}$ ), combining two well known probabilistic approaches: the Kalman Filter and Hidden Markov models. In this work we implemented the procedure shown in [8] using MatLab (The MathWorks,Inc.,Natick,MA,USA).

\section{Translating Standard Definitions to SLDS}

In this section we describe the correspondence of the standard definitions for movement analysis to the elements of the SLDS modelling. As an example we will use the knee angular displacement. Figure 1 shows a typical curve captured in our experiments for a Sit-Stand-Sit sequence. To completely describe the sit-stand-sit motion other variables, such as trunk tilt, are usually combined to the knee angular displacement. But since the goal of this paper is to show the application of the SLDS approach to segment human motion, for simplicity we use the knee angular displacement.

According to the definitions from Section II-A we have four events $\left(e_{1}, e_{2}, e_{3}, e_{4}\right)$, which are marked with arrows. In consequence there are four components, which physically correspond to Sit $\left(c_{1}\right)$, Knee Extension $\left(c_{2}\right)$, Stand $\left(c_{3}\right)$, Knee Flexion $\left(c_{4}\right)$. We can group the components in phases: the rising phase is composed by $c_{1}, c_{2}$ and the descending phase by $c_{3}, c_{4}$. A complete movement cycle consists of one rising and one descending phase.

In terms of SLDS elements, the four components correspond to four possible states for the switching variable, or $S=\left\{c_{1}, c_{2}, c_{3}, c_{4}\right\}$. The four events correspond to a change in the value of the switching variable. Modelling of the knee angle's dynamics during each component is done by four different state-space models, $\left(A_{i}, Q_{i}\right)$. The interaction between these models is governed by the switching state's transition matrix $\Pi$. Note that the dynamic behaviour in the first component $\left(\mathrm{Sit}, c_{1}\right)$ is similar to the third component (Stand, $c_{3}$ ). However, they differ because one is proceeded by a knee extension and the other by knee flexion. This condition is incorporated in the transition matrix $\Pi$. A physical interpretation is that it is impossible to immediately move from a standing position to sit, without performing a knee extension.Using the dataset presented in Figure 1 we learned the parameters for the SLDS to represent the sit-stand-sit movement. In this dataset the periods of the knee extension and knee flexion components are within the average for healthy adults shown in [10]. Besides estimating in which state the switching variable is, we also have an estimation of the continuous values of the knee angular displacement (the $x_{t}$ in equation 1). This information is not the focus of the present work, but it is certainly a useful feature for future applications.

\section{Experiments}

To show the advantages of the SLDS approach we compared it to an approach based on heuristics and threshold classification, represented as a finite state machine (FSM) model with transition thresholds based on the instant velocity of the knee displacement. As in the SLDS model, the dataset presented in Figure 1 was used to extract the FSM model's parameters. The simplest approach would be to set the threshold to zero during the sit and stand component, positive velocity for the knee extension component and negative velocity of the knee flexion. Looking closely to the instantaneous velocity shown in Figure 1, we see that these values are not suitable because the velocity is not constant during each component. This can be due to noise in the sensor or small movements which are captured by the sensors. As we will show in the results section, this thresholds are extremely dependent on the sample dataset 


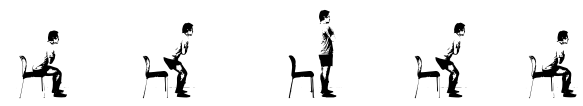
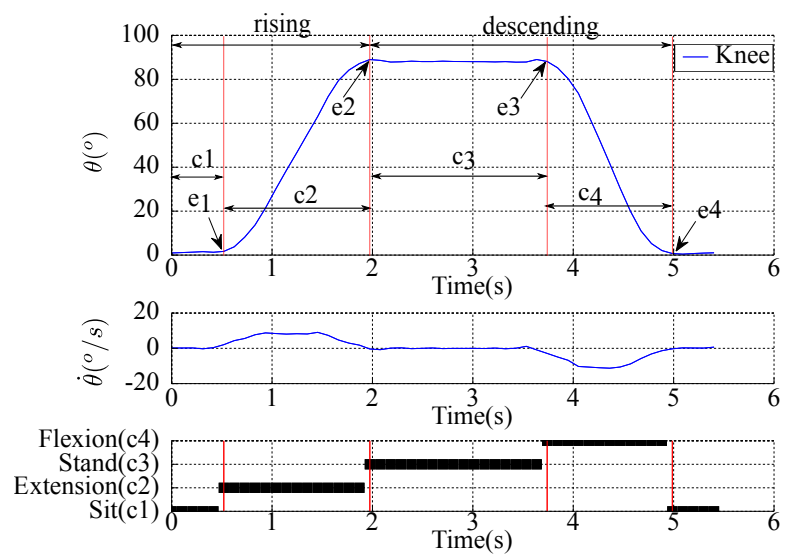

Fig. 1. Training dataset consisting of one execution of the Sit-Stand-Sit movement cycle. Events $\left(e_{i}\right)$, components $\left(c_{i}\right)$ and the rising and descending phases are marked. $\theta$ and $\theta$ indicates angle and angular velocity.

used for modelling. Other heuristic approaches can be used to extract a different model, but again they will be dependent on the dataset used for modelling and the variable in question [3].

Both models are crossvalidated in six scenarios: a single execution of the Sit-Stand-Sit movement executed with three different velocities, resulting in a "fast", "normal" and "slow" movement; a sequence of 5 consecutive Sit-StandSit, in which the subject was instructed to execute the knee extension and knee flexion at his self-selected velocity; and a sequence of 5 consecutive Sit-Stand-Sit movements in which the subject was instructed to execute each repetition at a randomly different velocity.

All datasets were recorded from the same subject (healthy, male, 32 years-old).

\section{RESULTS}

The results for the movement cycle diagram for one repetition, executed at different velocities is shown in Figure 2. For the first case, the "fast" execution, both approaches had similar results. in fact the finite state machine (FSM)/threshold approach was more accurate in detecting the transitions, matching the ground truth at $t=0.10 s$ (transition $c_{1} \rightarrow c_{2}$ ) and at $t=1.14$ (transition $c_{2} \rightarrow c_{3}$ ). The switching linear dynamical system (SLDS) model correctly estimated the sequence of components, with a delay of one sample in the events $c_{2} \rightarrow c_{3}$ and $c_{3} \rightarrow c_{4}$.

In the second case, the "normal" velocity execution (which is similar to the training data), the performance of the FSM/threshold approach is poor. It estimates early $c_{1} \rightarrow c_{2}$ (at $t=0.31 s$ ). The transition $c_{2} \rightarrow c_{3}$ is correctly estimated. But during component $c_{3}$ there is an incorrect estimation of $c_{3} \rightarrow c_{4}$, at $t=3.12 \mathrm{~s}$, which leads to a cycle through $c_{4} \rightarrow c_{1} \rightarrow c_{2} \rightarrow c_{3}$, anticipating the correct $c_{3} \rightarrow c_{4}$ transition at $t=3.53 \mathrm{~s}$. Again the SLDS approach estimated the events with one sample delay and correctly maintained the estimation throughout the component.

Finally, in the "slow" execution the FSM/threshold approach estimated the $c_{1} \rightarrow c_{2}$ transition very early on, at $t=0.10 \mathrm{~s}$. It then lead immediately to the estimation of transition $c_{2} \rightarrow c_{3}$, and remained on $c_{3}$ until $t=2.80 \mathrm{~s}$, when it estimated $c_{3} \rightarrow c_{4}$ at $t=2.91$. It then lead to a cycle through $c_{4} \rightarrow c_{1} \rightarrow c_{2} \rightarrow c_{3}$, returning to the correct component. The SLDS approach correctly estimated the sequence of events, with two samples delay in the transition detection at $t=0.83 \mathrm{~s}$, and one sample delay at $t=2.91 \mathrm{~s}$ and $t=5.19 \mathrm{~s}$.
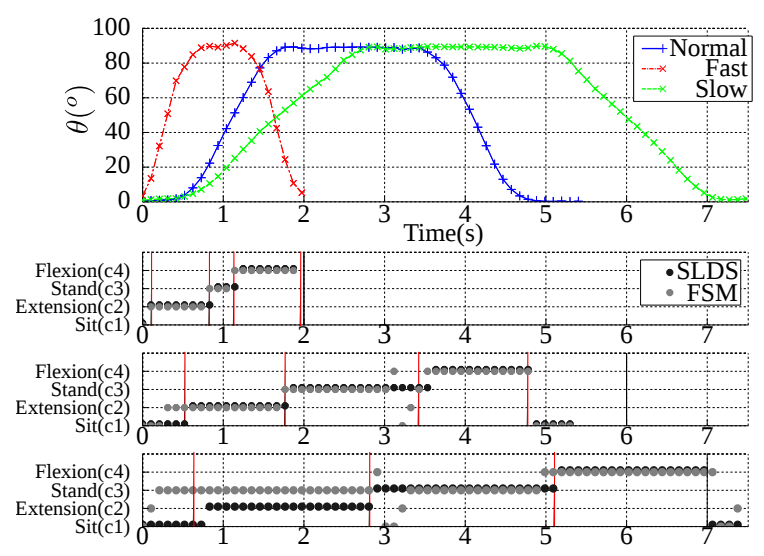

Fig. 2. Cross validation for the movement cycle extraction with the Switching Linear Dynamic System(SLDS) model and the Finite State Machine with thresholds (FSM) model using datasets containing one movement execution with different velocities: Normal, Fast and Slow. Red vertical lines represent the beginning of each component in the manually segmented dataset (used as ground truth).

In the 5 times Sit-Stand-Sit with "normal" velocity, presented in Figure 3, the FSM/Threshold approach exhibited the same misclassification issues seen on the cross validation with one repetition. Missed estimation of the transitions lead to cycle through before returning to the correct estimation at $t=3.43 \mathrm{~s}$ and at $t=16.72 \mathrm{~s}$. Again a premature estimation $c_{1} \rightarrow c_{2}$, at $t=23.50 \mathrm{~s}$ lead to an incorrect estimation of $c_{2} \rightarrow c_{3}$ at which the estimation is locked until it transits to another cycle through $c_{3} \rightarrow c_{4} \rightarrow c_{1} \rightarrow c_{2} \rightarrow c_{3}$, at $t=25.69 \mathrm{~s}$ and returns to the correct estimation path. The SLDS approach correctly estimated the sequence of events and had minor delays in the transition detections.

Figure 4 shows the last experiment, the 5 times SitStand-Sit with varied velocity. The same issues for the FSM/Threshold approach can be noticed again in this test: at $t=9.15 \mathrm{~s}$ and at $t=11.02 \mathrm{~s}$. The SLDS again correctly estimated the sequence of components, with some delay in the transition detection. Particularly at $t=15.08 \mathrm{~s}$, the SLDS was able to detect a $c_{4} \rightarrow c_{2}$ transition. In this case, since it is just a valley point, there was no consecutive samples at the "Sit" (or component $c_{1}$ ). The FSM/Threshold had to go through component $c_{1}$, in order to reach the correct estimation of $c_{2}$. 

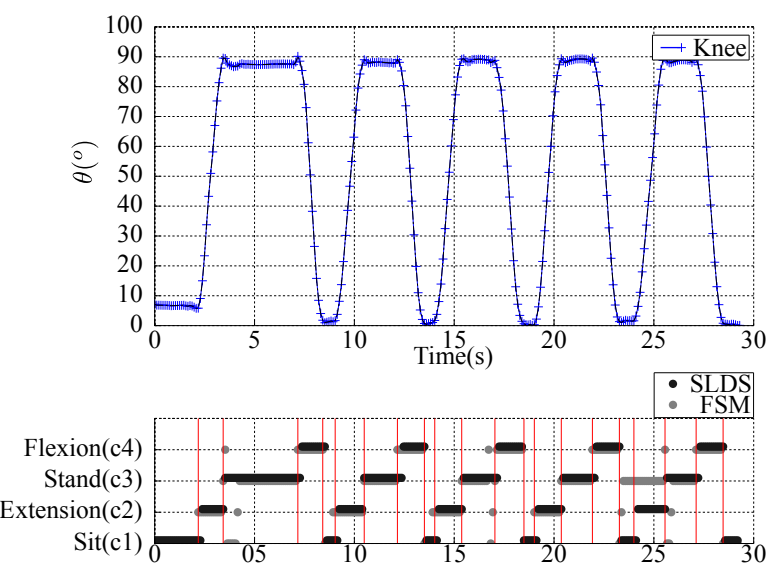

Fig. 3. Cross validation for the movement cycle extraction with the Switching Linear Dynamic System(SLDS) model and the Finite State Machine with thresholds (FSM) model using datasets containing a sequence of 5 Sit-Stand-Sit movements executed with normal velocity. Red vertical lines represent the beginning of each component in the manually segmented dataset (used as ground truth).
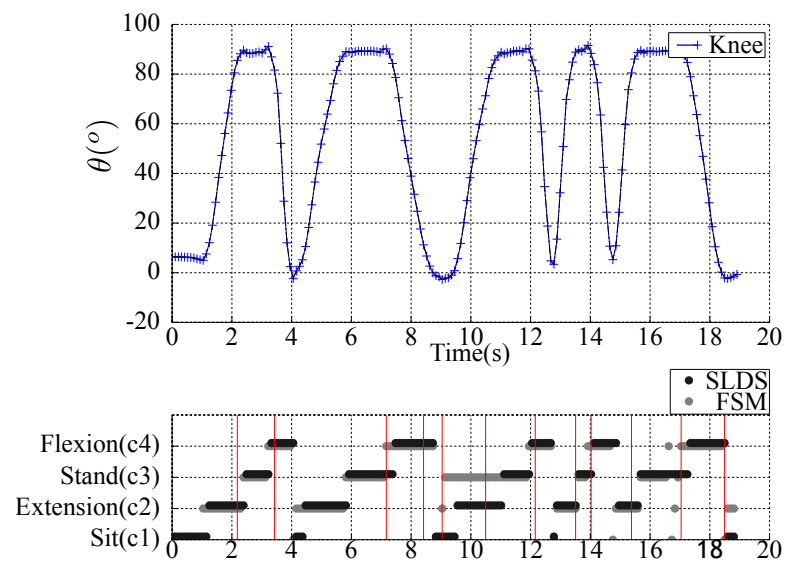

Fig. 4. Cross validation for the movement cycle extraction with the Switching Linear Dynamic System(SLDS) model and the Finite State Machine with thresholds (FSM) model using datasets containing a sequence of 5 Sit-Stand-Sit movements executed with varied velocity. Red vertical lines represent the beginning of each component in the manually segmented dataset (used as ground truth).

\section{DISCUSSION}

We showed that the modelling of the Sit-Stand-Sit motion converting the standard definitions into elements of the SLDS model results in an efficient and robust model to segment and extract useful information, i.e. spatiotemporal features, and generate the movement cycle diagram. The results support that our approach is a straight-forward modelling procedure, requires a small training dataset and is suited for classifying and segmenting the components of a movement.

In the current stand we presented results for a univariate case (only the knee angle was used). A more complete description of the Sit-Stand-Sit motion, or other movement types, such as gait, requires a combination of multi-joint variables. The procedures and algorithms used here are suited for the multivariate case, but we kept the initial investigation to the univariate case. Further work is required to evaluate the performance of the multivariate case.
Furthermore, the SLDS approach provides us more information than we presented in this paper, such as the a priori and posterior probabilities of $s_{t}$ and $x_{t}$ in Equation 1. In future works all this information can be used not only to segment a known movement type for spatiotemporal feature extraction, but also to distinguish between two different motions, similar to the work presented by [5].

This result shifts from the heuristics based or custom build algorithms which are strongly dependent on the dataset or the movement studied. Since we strongly based our approach on the standard definitions of the movement, the information extracted can readily be compared to standardized results for healthy and impaired subjects such as shown in [1], [11].

\section{CONCLUSIONS}

In this paper we presented a straight forward procedure for automatic extraction of spatiotemporal features and generation of a movement cycle diagram combining the standard definitions from movement analysis and switching linear dynamic system modelling. The results for the Sit-StandSit example demonstrate that the resulting model has a good performance to classify a known movement type executed in various ways. The next step is to investigate the performance of this methodology for the multivariate case and across different populations.

\section{REFERENCES}

[1] K. Kerr, J. White, D. Barr, and R. Mollan, "Analysis of the sit-stand-sit movement cycle in normal subjects," Clinical Biomechanics, vol. 12, no. 4, pp. 236-245, June 1997.

[2] T. A. L. Wren, G. E. Gorton, S. Ounpuu, and C. A. Tucker, "Efficacy of clinical gait analysis: A systematic review." Gait \& posture, vol. 34, no. 2, pp. 149-53, June 2011.

[3] N. Millor, P. Lecumberri, M. Gomez, A. Martinez-Ramirez, and M. Izquierdo, "Kinematic Parameters to Evaluate Functional Performance of Sit-to-Stand and Stand-to-Sit Transitions Using Motion Sensor Devices: A Systematic Review." IEEE Transactions on Neural Systems and Rehabilitation Engineering, vol. PP, no. 99, p. 1, June 2014.

[4] H. Zhou and H. Hu, "Human motion tracking for rehabilitation - A survey," Biomedical Signal Processing and Control, vol. 3, no. 1, pp. 1-18, Jan. 2008.

[5] J. F.-s. Lin and D. Kulic, "Online Segmentation of Human Motion for Automated Rehabilitation Exercise Analysis," IEEE Transactions on Neural Systems and Rehabilitation Engineering, vol. 22, no. 1, pp. 168-180, 2014.

[6] P. de Dios, P. Chung, and Q. Meng, "Landmark-Based Methods for Temporal Alignment of Human Motions," IEEE Conputational Intelligence Magazine, no. April 2014, pp. 29-37, 2014.

[7] W. Tao, T. Liu, R. Zheng, and H. Feng, "Gait analysis using wearable sensors." Sensors (Basel, Switzerland), vol. 12, no. 2, pp. 2255-83, Jan. 2012.

[8] V. Pavlovi, J. M. Rehg, T.-j. Cham, and K. P. K. Murphy, "A dynamic Bayesian network approach to figure tracking using learned dynamic models," in Proceedings of the Seventh IEEE International Conference on Computer Vision, vol. 1, no. Iccv 99. IEEE, 1999, pp. 94-101 vol.1.

[9] Z. Ghahramani and G. E. Hinton, "Variational Learning for Switching State-Space Models," Neural Computation, vol. 12, no. 4, pp. 831864, Apr. 2000.

[10] K. Kerr, J. White, D. Barr, and R. Mollan, "Standardization and definitions of the sit-stand-sit movement cycle," Gait \& Posture, vol. 2, no. 3, pp. 182-190, Sept. 1994.

[11] O. Sofuwa, A. Nieuwboer, K. Desloovere, A.-M. Willems, F. Chavret, and I. Jonkers, "Quantitative gait analysis in Parkinson's disease: comparison with a healthy control group." Archives of physical medicine and rehabilitation, vol. 86, no. 5, pp. 1007-13, May 2005. 